\title{
CURRENT MANAGEMENT OF ACUTE MENISCAL INJURIES
}

\author{
Paul Marshall, Consultant Orthopaedic Surgeon \\ Lancaster \& Kendal Hospitals
}

\section{INTRODUCTION - FUNCTIONS OF THE MENISCI AND MECHANISMS OF MENISCAL INJURY}

The arrangement of the collagen bundles within the meniscus appears to be ideal for the absorption and translation of vertical compression loads into circumferential stresses. The menisci distribute forces across the joint by increasing the contact area between the femoral condyles and the tibial plateaux. Studies have shown that the menisci take between 50 and $70 \%$ of the total knee load. Specifically, $90 \%$ of the load across the knee joint passes through the medial compartment, and $50 \%$ of this is taken by the medial meniscus. Ten per cent of the total load across the knee joint passes through the lateral compartment, of which $70 \%$ is taken by the lateral meniscus. Menisectomy dramatically alters the pattern of static loading across the knee joint, and several studies have demonstrated greater stress concentration and decreased shock-absorbing capabilities after total menisectomy.

In childhood, the meniscus is white, translucent, and has an abundant blood supply. With ageing, the vascularity of the meniscus decreases and it becomes brittle and yellowish in colour. The meniscus of the sportsperson is 'at risk' because it is at the stage when it is undergoing degeneration from translucent white to opaque yellow. When the shear stress within the meniscus exceeds the tissue strength, a tearing injury results. Should an excessive force be applied to a normal meniscus, then a vertical tear is likely, which is the more common type of tear seen in sporting injuries. Should a normal force be applied to a degenerate meniscus, then a horizontal cleavage tear is more likely. When the femur is internally rotated on a flexed knee the posterior horn of the medial meniscus may become trapped beneath the medial femoral condyle, and a tear may result if the joint is suddenly extended. Similarly, when the femur is laterally rotated on a flexed knee the posterior horn of the lateral meniscus may become trapped beneath the lateral femoral condyle, and a tear may result if the knee is suddenly extended. Medial meniscal injuries are seen up to five times more commonly than lateral meniscal injuries in most sports. This is related to the higher incidence of 'side-on' tackles which apply a valgus strain to the knee. Owing to the valgus nature of these injuries, there is often a concomitant medial collateral ligament injury (Fig 1).

\section{DIAGNOSIS OF MENISCAL INJURIES - HISTORY}

Almost all younger patients will be able to recall some type of twisting or overloading episode. Some patients may continue their activity, while others may find it too painful to

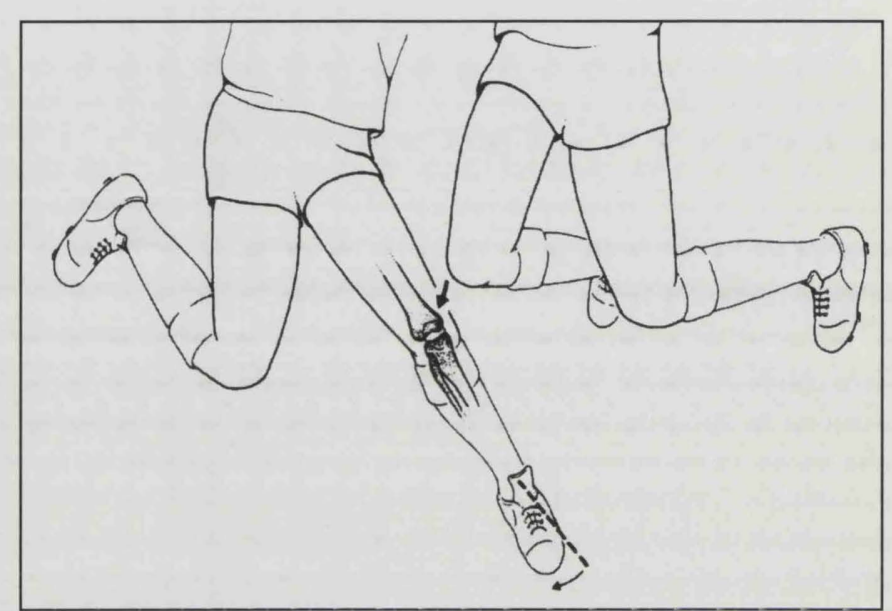

Fig 1 - The side-on tackle applies a valgus knee strain which may cause meniscal injury as well as medial collateral ligament injury

bear weight on the affected knee. The difficulty with movement is usually pain-related, although in a small number of patients this difficulty will be due to true locking secondary to a displaced torn meniscal fragment.

Patients with an acute meniscal tear usually develop a gradual, mild knee effusion over a period of approximately twelve hours. This is in contrast to patients who sustain a haemarthrosis, such as following an injury to the anterior cruciate ligament, who develop a large swelling of the knee immediately following the injury.

A number of patients, usually between the ages of 40 and 60 years, present with symptoms suggestive of meniscal pathology, but there is no definite history of either specific twisting injury or overloading. Many of these will have developed their symptoms of pain and swelling over the previous weeks or months and some may recall experiencing their first episode of joint pain while squatting or while ascending from a squatting or sitting position. These patients are likely to have sustained a horizontal cleavage tear in a degenerate meniscus, and radiographs may show evidence of degenerative joint disease.

\section{DIAGNOSIS OF MENISCAL INJURIES - CLINICAL SIGNS}

\section{Joint-line tenderness}

Joint-line tenderness is usually a reliable indicator of meniscal injury. Tenderness above or below the joint line may indicate the presence of a concomitant collateral ligament sprain.

\section{Swelling}

Effusions associated with meniscal tears are usually mild. The patellar tap sign may not be present, but a visible ripple 
of fluid in the parapatella fossa may be demonstrated by first expressing fluid from one side of the joint and the suprapatella pouch, and then gently stroking the opposite side of the joint with the palm of the hand.

\section{Tibial rotation tests}

Tibial rotation tests have been described by McMurray ${ }^{(1)}$ and Apley ${ }^{(2)}$ and were devised in attempts to differentiate a torn meniscus from other knee pathology. Both tests aim to trap or catch the torn meniscus between the femoral condyle and the tibial plateau. A positive rotation test is associated with either a palpable or audible click which may reproduce the patient's painful symptoms. These tests are helpful when positive, but not when negative, and may be difficult to perform on an acutely painful knee. It is important to perform the rotation test on the opposite knee, as similar clicking may also be present. The McMurray manoeuvre is performed by externally rotating the leg with the knee fully flexed (Fig 2). A varus stress is then applied as the knee is gradually extended. A palpable, audible or painful click may indicate a tear of the posterior horn of the medial meniscus. The manoeuvre is then repeated with forcible internal rotation, while applying a valgus stress, to elicit postero-lateral meniscal clicks.

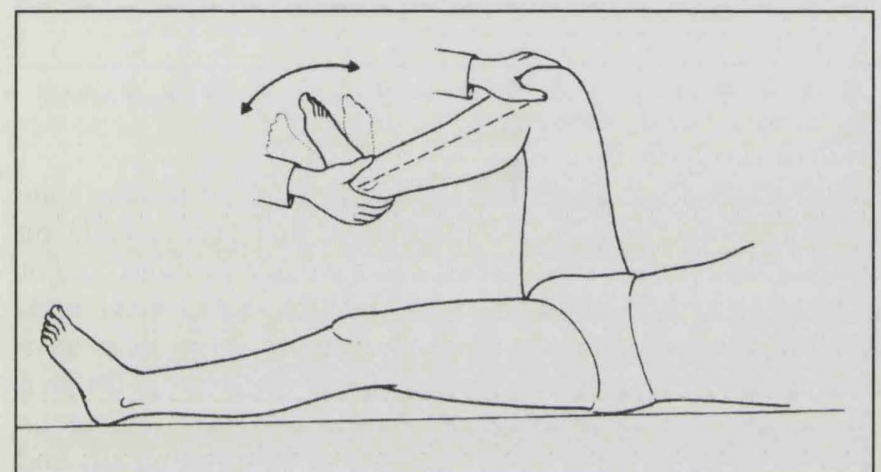

Fig 2 -McMurray's tests: the knee is extended, applying a varus stress with external rotation (medical meniscus) and then applying a valgus stress with internal rotation (lateral meniscus)

The Apley grinding test is performed with the patient in the prone position. The examiner rotates the flexed tibia on the femur while exerting downward pressure along the long axis of the tibia. With pressure exerted during rotation, pain may be felt in the region of a torn meniscus.

In addition to the tibial rotation tests, asking the patient to squat or duck-walk may reproduce joint-line pain if a meniscal tear is present.

\section{MENISCAL CYSTS}

Meniscal tears may occur in association with cysts of the menisci. These are more commonly seen on the lateral side and are invariably associated with a radial or horizontal cleavage tear of the lateral meniscus. Patients usually present with pain in the lateral joint-line region which persists for a number of hours following activity. Most patients are conscious of a small swelling on the lateral joint-line which fluctuates in size. Examination will reveal a tender palpable mass on the lateral joint-line which is maximally distended at approximately 30 degrees of flexion. The differential diagnosis is that of a ganglion, an osteophyte in an arthritic knee, or a small soft tissue tumour such as a synovial cell sarcoma. Meniscal cysts were traditionally treated by total menisectomy and open removal of the cyst. Currently, these cysts are now effectively treated by arthroscopic resection of the tear, allowing decompression of the cyst, and it is not necessary to remove the cyst through a separate skin incision.

\section{DIAGNOSTIC ADJUVANTS - MRI AND ARTHROSCOPY}

\section{Magnetic resonance imaging (MRI)}

Owing to its remarkable soft tissue resolution, MRI has become an important diagnostic tool in the evaluation of lesions of the menisci. A negative predictive value for the diagnosis of a torn meniscus is around $90 \%$, which means that $10 \%$ of the time a negative finding on the MRI study may hide the presence of a torn meniscus. MRI has the advantage of being noninvasive, but has the disadvantages of being time consuming and expensive. We would not routinely advocate the use of MRI for evaluation of meniscal tears. If the clinical history and physical signs suggest meniscal pathology, arthroscopy is indicated. MRI is only indicated if a diagnosis is unclear and when it is felt that the treatment of the patient would be affected by the results.

\section{Arthroscopy}

The advent of arthroscopy of the knee over the past 10 to 15 years has revolutionised the diagnosis and treatment of meniscal tears. It allows visualisation and direct palpation of the various intra-articular structures. A probe can be used to assess the integrity of the medial and lateral menisci, the anterior cruciate ligament, and the status of the articular surfaces of the femoral condyles, tibial plateaux, and patella, as well as the synovial lining of the joint. The widespread use of arthroscopy has eliminated the necessity for exploratory arthrotomies and enables meniscal surgery to be performed as an outpatient procedure. The surgery can be performed under general or spinal anaesthesia and is usually associated with minimal pain, allowing patients to walk immediately following surgery without the necessity for crutches. Rehabilitation is usually rapid, allowing patients to return to work within days and to sporting activity within two to three weeks. In addition to the clinical benefits, there are significant economic advantages in terms of containment of hospital costs, absence from work, and early return to sport.

\section{Indications for arthroscopy}

Diagnosis and treatment of meniscal tears is the main indication for arthroscopy of the knee. However, not every knee requires an arthroscopy. Patients presenting with typical symptoms of joint-line pain and swelling, with or without episodes of locking, are usually good candidates for arthroscopy. Such patients may have positive clinical signs, including joint-line tenderness, effusion within the joint, and meniscal catching signs. Arthroscopy may also be considered for those patients who have persistent symptoms suggestive of a meniscal tear, despite the absence of clinical signs. Prudent clinical judgement is paramount. If symptoms are vague and diffuse, the success rate from arthroscopy is similarly reduced. Arthroscopy should not be considered unless there is a reasonable chance of providing benefit to the patient, either by providing more information than was known clinically, or by providing treatment. Performing arthroscopy on patients who have little chance of gaining from the procedure only serves to increase their expectations, which in turn will lead to further disappointment.

\section{CURRENT MANAGEMENT OF ACUTE MENISCAL INJURIES}

Knowledge of the consequences of total menisectomy and the functional role of the meniscus in force transmission and shock absorption has led to the principle of preserving as much functional meniscus as possible. In partial menisectomy, meniscal resection is confined to the loose 
unstable fragment, such as the displaceable inner edge of a bucket handle tear or the flap in an oblique tear. Following partial menisectomy, a stable or balanced rim of healthy meniscal tissue is preserved. This intact balanced peripheral rim provides joint stability and protects the articular surfaces by its load-bearing functions. Preservation of this peripheral cartilage rim is particularly important in patients who have already had major ligamentous injuries.

Many studies have shown that there is an increased incidence of degenerative change following total, as opposed to partial menisectomy, and this has led to the perceived need to consider repairing torn menisci. Several studies have documented that the peripheral one third of the meniscus has a vascular supply, and that tears in this zone have a potential to heal. There is greater penetration of the vasculature in the skeletally immmature individual, and thus children have a greater potential for the healing of meniscal tears.

\section{ARTHROSCOPIC MANAGEMENT}

Arthroscopic management of the torn meniscus is now standard, and has virtually replaced arthrotomy and open menisectomy. Arthroscopy affords much better exposure than can be gained by arthrotomy. Tear patterns can be readily identified and explored, following which appropriate management strategies can be planned. Nowadays, total menisectomy is very rarely required, and common current management strategies for meniscal tears include partial menisectomy, meniscal repair, and the most conservative treatment of all, which is to leave the tear alone. Tears which are suitable for conservative management are partial thickness split tears and short, full thickness radial tears which are stable on meniscal probing.

\section{PARTIAL MENISECTOMY}

Meniscal tears which are not suitable for repair because they are located within the avascular zone, or because of the extent of meniscal substance damage, are treated by partial menisectomy. When partial menisectomy is performed, only the unstable offending segment is removed, leaving behind an intact peripheral rim which maintains stability and protection to opposing articular surfaces (Fig 3). Once the decision is made to resect the torn mobile part of the meniscus, it is removed either en bloc (Fig 4) or by morcellization (piecemeal) with a basket forceps. Following removal of the obviously mobile torn fragment, a further check of the remaining meniscus is made to ensure that the rim is balanced and stable.

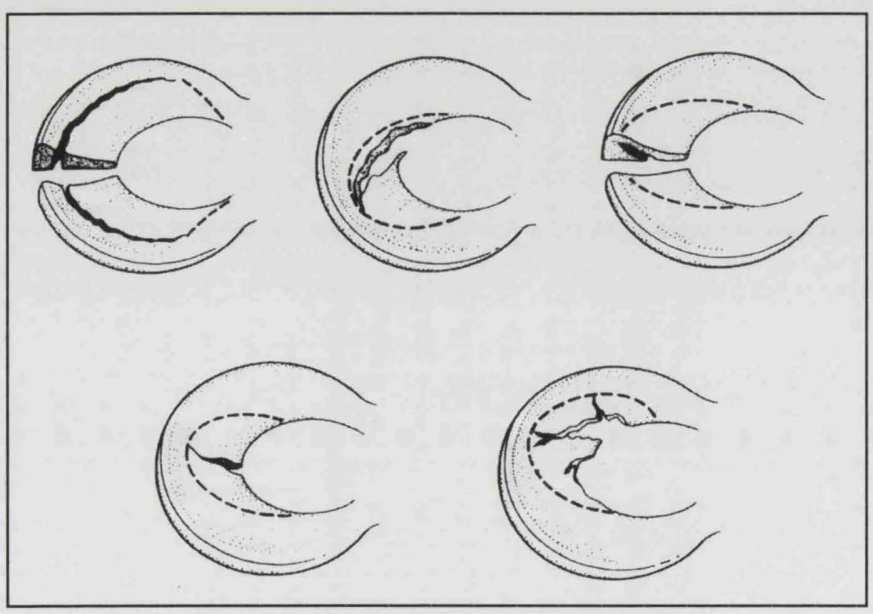

Fig 3 - Partial menisectomy resection lines

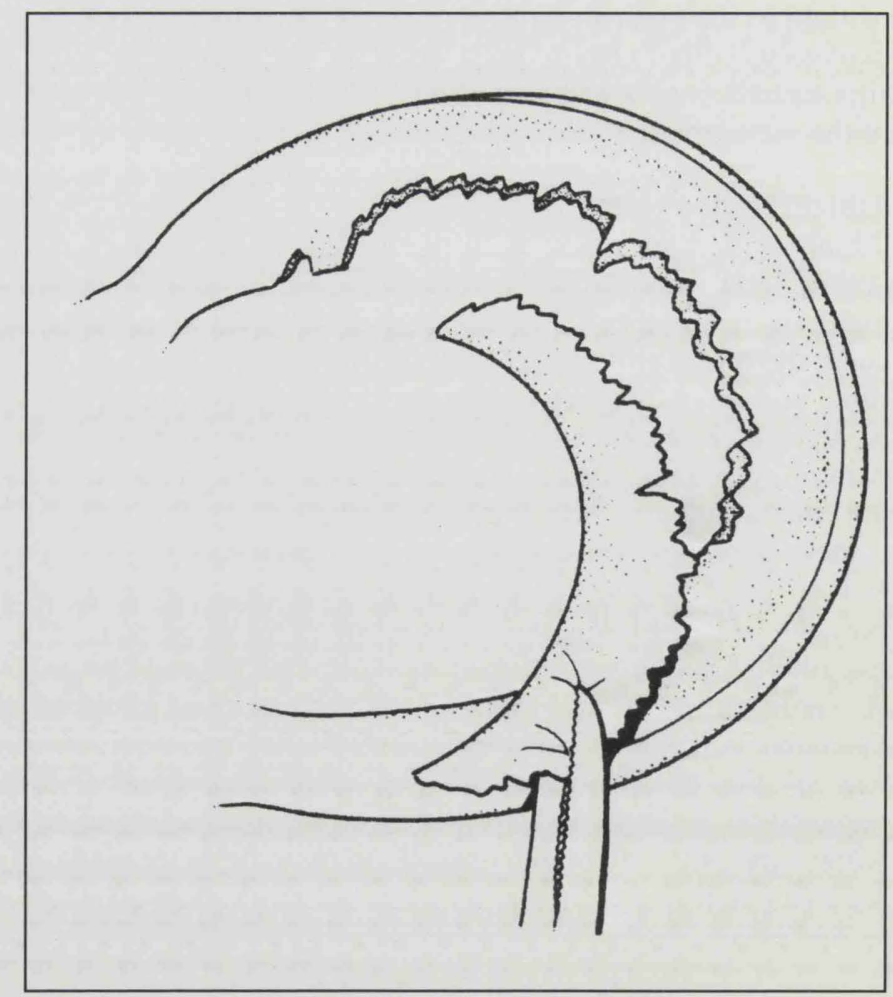

Fig 4 - Partial menisectomy: removal of the mobile torn fragment en bloc

\section{Meniscal repair}

Tears suitable for repair are those within the vascular zone (the outer one third of the meniscus), that are unstable on probing, are longer than $7 \mathrm{~mm}$, and have not sustained major damage. It is frequently observed that tears which have been displaced into the intercondylar notch for some time have shortened and cannot be properly repositioned to the original rim of the torn meniscus. Such tears which have undergone alteration in size and shape are not suitable for repair, and should be resected.

Repair can be performed by open direct suture of tears which are at the very periphery of the meniscus, or by using

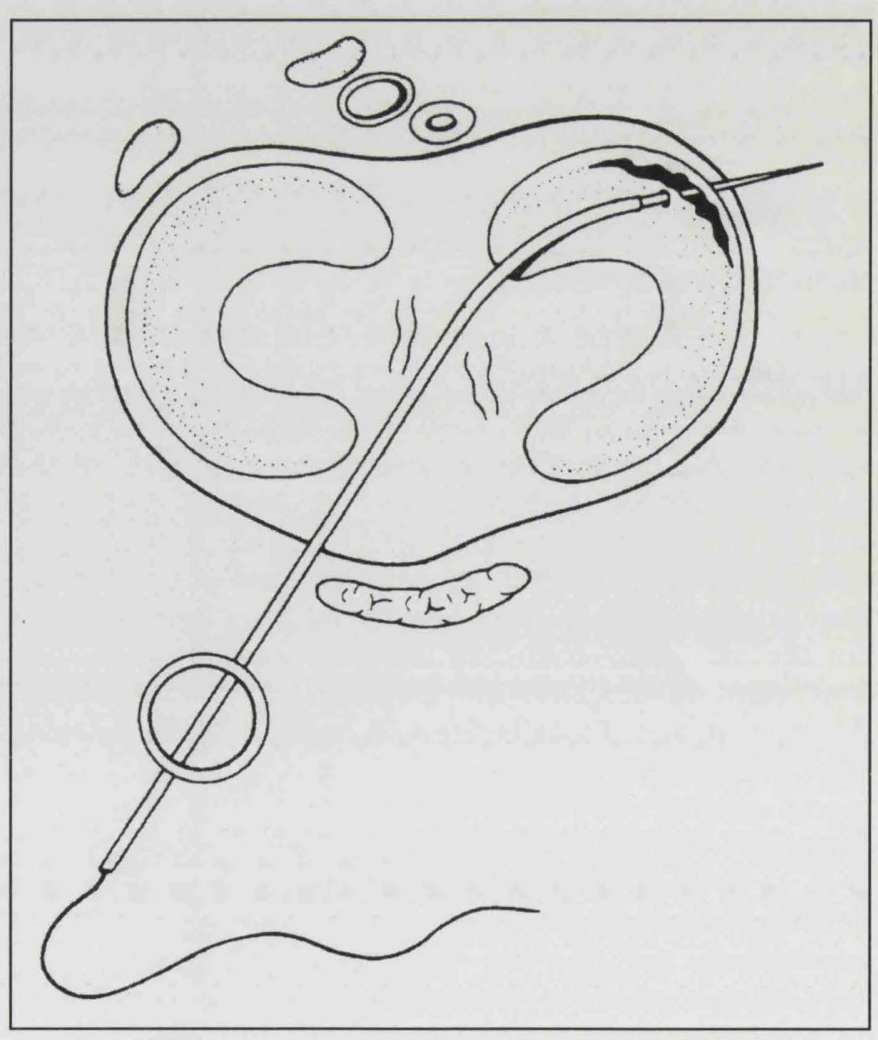

Fig 5 -Meniscal repair. A curved cannula is introduced through the contralateral arthroscopy portal to avoid the popliteal vessels and nerve 
variously described arthroscopic techniques (Fig 5). Traumatic tears within the vascular zone have healing rates of up to $90 \%$ whether the repairs are performed by open or arthroscopic methods. Acute tears, of less than two months' duration, have significantly greater rates of healing following repair than chronic tears seen after many months. When a tear suitable for repair is seen in a knee with a deficient anterior cruciate ligament, this ligament should be reconstructed at the time of meniscal repair, otherwise there is a high likelihood that the meniscal repair will fail.

\section{REHABILITATION FOLLOWING MENISCAL SURGERY}

Isometric quadriceps exercises are started as soon as the patient returns to the day surgery unit and early active movements are encouraged. Full weight bearing is allowed as soon as the patient feels comfortable and a full range of knee movement should be restored by five to seven days. Early return to work is encouraged, for example after two to three days for patients with sedentary occupations, and after one to two weeks for patients having heavier work. Sporting activities are allowed when there is a full range of knee motion, $75 \%$ quadriceps strength, no pain, and minimal effusion. This usually takes two to four weeks. Postoperative effusions usually resolve over a period of two to three weeks, and tend to resolve more quickly in patients who have undergone medial, rather than lateral meniscal surgery.

Rehabilitation programmes following meniscal repair involve an early return to knee movements, but weight bearing is usually restricted for around eight weeks postoperatively.

\section{REFERENCES}

1 McMurray TP The semilunar cartilages Br J Surg 1941; 29(116):407-14

2 Apley AG The diagnosis of meniscal injuries: some new clinical methods JBJS 1947; 29:78-84

Raymond Carver (1939-1988) wrote this poem and the one on page 344 when he was dying of cancer. He was an alcoholic who met and married the American poet and short story writer Tess Gallagher ten years before his death. He dried out and entered a sustained creative burst of writing which was nourished by the deep love they shared. His contentment and humility are plain to see and it is remarkably that his pulmonary tumour had already metastasized to his brain when he wrote these poems. He died very shortly afterwards.

\section{Gravy}

No other word will do. For that's what it was. Gravy.

Gravy, these past ten years.

Alive, sober, working, loving and

being loved by a good woman. Eleven years

ago he was told he had six months to live

at the rate he was going. And he was going

nowhere but down. So he changed his ways

somehow. He quit drinking! And the rest?

After that it was all gravy, every minute

of it, up to and including when he was told about,

well, somethings that were breaking down and

building up inside his head. 'Don't weep for me',

he said to his friends. 'I'm a lucky man.

I've had ten years longer than I or anyone expected. Pure gravy. And don't forget it.' 